\title{
Hepatitis E Virus Capsid as a Carrier of Exogenous Antigens for the Development of Chimeric Virus-Like Particles
}

\author{
Tianyu Lu ${ }^{a} \quad$ Nouredine Behloul ${ }^{b} \quad$ Yi Zhou $^{a}$ Sarra Baha ${ }^{a} \quad$ Zhenzhen Liu ${ }^{a}$

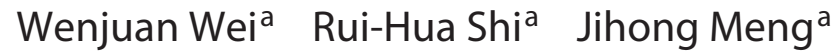 \\ aDepartment of Gastroenterology, Zhongda Hospital, Southeast University, Nanjing, China; ${ }^{b}$ College of Basic \\ Medicine, Shanghai University of Medicine \& Health Sciences, Shanghai, China
}

\section{Keywords}

Hepatitis E virus · Exogenous antigen carrier · Virus-like particle $\cdot$ Immunogenicity

\begin{abstract}
Introduction: Virus-like particles (VLPs), self-assembled multiprotein structures, can stimulate robust immune responses due to their structural similarity to native virions that allow the presentation of multiple copies of the target epitopes. Utilizing VLPs as vaccine platforms to present exogenous antigens is a promising and challenging approach in the vaccine development field. This study investigates the potential of the truncated hepatitis E virus (HEV) capsid as a VLP platform to present foreign antigens. Methods: The S and M domains of the HEV capsid protein were selected as the optimal carrier (CaSM). The exogenous antigen Seq8 containing 3 neutralizing epitopes from 3 different foot-and-mouth disease virus (FMDV) strains was linked to the C-terminal of CaSM to construct a chimeric VLP (CaSM-Seq8). The chimeric particles were produced in Escherichia coli, and their morphology, physicochemical properties, antigenicity, and immunogenicity were analyzed. Results: Morphological analysis showed that CaSM-Seq8 self-assembled into VLPs similar
\end{abstract}

karger@karger.com www.karger.com/int

Karger $\stackrel{\text { ' }}{5}$

GOPEN ACCESS
C 2021 The Author(s)

Published by S. Karger AG, Basel

This is an Open Access article licensed under the Creative Commons Attribution-NonCommercial-4.0 International License (CC BY-NC) (http://www.karger.com/Services/OpenAccessLicense), applicable to the online version of the article only. Usage and distribution for commercial purposes requires written permission. to CaSM VLPs ( $26 \mathrm{~nm}$ in diameter) but smaller than native $\mathrm{HEV}$ virions. Further, the thermal stability and the resistance to enzymatic proteolysis of Seq 8 were enhanced when it was attached to the CaSM carrier. The antigenicity analysis revealed a more robust reactivity against anti-FMDV antibodies when Seq8 was presented on CaSM particles. Upon injection into mice, FMDV-specific lgGs induced by CaSM-Seq8 appeared earlier, increased faster, and maintained higher levels for a longer time than those induced by Seq 8 alone or the inactivated FMDV vaccine. Conclusion: This study demonstrated the potential of utilizing the truncated HEV capsid as an antigen-presenting platform for the development of chimeric VLP immunogens.

(C) 2021 The Author(s)

Published by S. Karger AG, Basel

\section{Introduction}

A virus-like particle (VLP) is a vacant self-assembled multiprotein structure that does not contain any viral genome $[1,2]$. VLPs can stimulate robust humoral and cellular immune responses because of their relatively large

Tianyu Lu and Nouredine Behlou contributed equally to this work.
Correspondence to:

Nouredine Behloul, nouredine_behloul@ hotmail.com

Ruihua Shi, ruihuashi@126.com

Jihong Meng, jihongmeng@163.com 
size similar to native virions and the presence of multiple copies of the target epitopes [3-5]. Given these unique characteristics, utilizing VLPs as platforms for the presentation of exogenous antigens becomes a promising approach for the development of effective recombinant vaccines $[3,6]$.

Hepatitis E virus (HEV) is an icosahedral spherical virus, with a diameter of $27-32 \mathrm{~nm}$, belonging to the Orthohepevirus genus of the Hepeviridae family $[7,8]$. The HEV capsid protein (encoded by the ORF2) contains 3 domains named: $S$ domain (residues 129-319), $\mathrm{M}$ domain (residues 320-455), and P domain (residues 456606). These 3 domains play different structural and functional roles in the HEV capsid [9]. The $S$ and $M$ domains form an internal scaffold shell connected to the $\mathrm{P}$ domain through a long proline-rich hinge. This latter allows the $\mathrm{P}$ domain to protrude from the surface of the HEV particles with certain flexibility for a better exposition of the dominant neutralization epitopes and the motifs critical for the interaction with the host-cell receptors. Therefore, such a structural assembly allows optimal exposure of the functional $\mathrm{P}$ domains as protruding spikes $[9,10]$. Previously, it has been reported that a truncated HEV ORF2 protein (aa 112-660) expressed in insect cells could form VLPs similar to the native HEV virions [11]. Further, the truncated HEV capsid protein also self-assembled into VLPs even with the $\mathrm{N}$-terminal truncation up to residue 125 and the C-terminal truncation up to residue 602 , indicating that the essential elements for the self-assembly were located between residues 125-602 [12], and the S domain was indispensable for the formation of $T=1$ particles [13]. However, later on, an N-terminal truncation up to aa367 has been reported as the largest truncation that maintained the self-assembly ability as it has been shown with the recombinant vaccine p239 [13, 14]. Considering the structural features and the ability of truncated HEV capsid proteins to maintain the self-assembly property, we investigated the use of the $\mathrm{S}$ and $\mathrm{M}$ domains as an antigen-presenting carrier (CaSM) by replacing the protruding $\mathrm{P}$ domain with an exogenous antigen.

Several antigen-presenting platforms based on the HEV capsid protein were previously designed by inserting foreign antigens at different positions: (1) inserting an 18 aa peptide of HIV-1 gp120 into the HEV capsid between residues 485 and 486 [6]; (2) inserting a myc-tag/ FLAG-tag/HA-tag between residue 488 and residue 489 to present multiple foreign antigens [15]; (3) adding a Bcell epitope tag consisting 11 amino acids to the $\mathrm{C}$-terminal [16]; (4) linking hepatitis A virus VP1 (aa 24-171) to the $\mathrm{C}$-terminal of the $\mathrm{P}$ domain to make a bivalent vaccine
[17]; and (5) fusing HEV ORF3 p70-123 to ORF2 p112608 [18]. However, in such designs, the inserted fragments were mere linear epitopes or too short which could not evoke potent immune responses, and some epitopes were attached to the $\mathrm{C}$-terminal of the $\mathrm{P}$ domain, which may restrict the flexibility and exposure of the exogenous antigens and/or the $\mathrm{P}$ domain [19]. Therefore, in this study, we aimed to overcome these drawbacks by selecting a large exogenous antigen, substituting the entire $\mathrm{P}$ domain, and linking it to the $\mathrm{M}$ domain through the HEV intrinsic proline-rich hinge.

Foot-and-mouth disease virus (FMDV), belonging to the genus Aphthovirus of the family Picornaviridae, is the causative pathogen of foot-and-mouth disease (FMD), an acute and contagious disease of clove-hoofed animals with devastating economic repercussions $[20,21]$. The genome of the FMDV encodes a large polyprotein that can be cleaved into 4 structural proteins (VP1-4) and several nonstructural proteins [20]. Seven FMDV serotypes have been identified: O, A, C, Asia 1, SAT 1, SAT 2, and SAT 3, with the serotype $\mathrm{O}$ as the predominant type in Asia; the serotype $\mathrm{O}$ can be further classified into 11 topotypes according to the characteristics of the VP1 protein $[20,22,23]$. Previous studies have revealed that $2 \mathrm{immu}-$ nogenic regions of VP1 aa 140-160 (G-H loop) and aa 200-213 (C-terminal fragment) can elicit neutralizing antibodies [24, 25]. Further studies demonstrated that although the G-H loop could elicit neutralizing antibodies, it had low immunogenicity because of its short length and the lack of T-cell epitopes [25]. The immunogenicity of the G-H loop could be enhanced drastically when it was incorporated with glutaraldehyde, liposome, and polyinosinic-cytidylic acid or when linked with the hepatitis B core protein, and such formulations also conferred complete protection in the immunized animals [26-28].

In the present work, we aimed to assess the truncated HEV capsid protein as a carrier of exogenous antigens. Such carrier (named CaSM) contains the S and M domain of the HEV capsid, and the foreign antigen would substitute the protruding HEV P domain, yielding a construct "S-domain M-domain exogenous antigens" (CaSMEAg). As a foreign antigen, we constructed the FMDV Seq 8 antigen by combining 3 FMDV G-H loops (aa 140160) obtained from the 3 most prevalent FMDV/O topotype strains in China: O/HN/CHA/09, O/IRN/2010, and $\mathrm{O} / \mathrm{Mya} / 98$. Then, we investigated whether the chimeric CaSM-Seq 8 could self-assemble into VLPs, presenting the Seq8 antigen on the surface and whether this combination would enhance the physical, biological, and immunological characteristics of the inserted antigen Seq8.
38

Intervirology 2022;65:37-48 DOI: $10.1159 / 000515719$
Lu/Behloul/Zhou/Baha/Liu/Wei/Shi/ Meng 


\section{Materials and Methods}

\section{Construction of the Target Clones}

The plasmid pET28-W2-1 containing the gene coding for the ORF2 protein of the HEV W2-1 isolate (GenBank: JQ655734.1) has been synthesized (Gene Create Co., Wuhan, China). The pET28-Seq 8 plasmid has been previously designed, synthesized, and stored in our lab. The polymerase chain reactions (PCRs) were performed using $2 \times$ Taq Master Mix (Vazyme Co., Nanjing, China) to amplify the target genes (NcoI-CaSM-BamHI, BamHI-Seq8XhoI, NcoI-CaSM-XhoI, and NcoI-Seq8-XhoI). The products NcoI-CaSM-BamHI and BamHI-Seq8-XhoI were digested by the BamHI restriction enzyme (Thermo Fisher Scientific Inc., Waltham, MA, USA) and linearly ligated by T4 DNA Ligase (Thermo Fisher Scientific Inc.) to construct NcoI-CaSM-Seq8-XhoI. Then, the ligated gene was further amplified by PCR.

Next, all the target genes (NcoI-CaSM-XhoI, NcoI-Seq8-XhoI, and NcoI-CaSM-Seq8-XhoI) were digested by NcoI and XhoI endonucleases (Thermo Fisher Scientific Inc.) and inserted into a linearized plasmid (NcoI-pET28a-XhoI) to construct the recombinant plasmids: pET28a- ${ }_{\mathrm{NcoI}} \mathrm{CaSM}_{\mathrm{XhoI}}, \mathrm{pET} 28 \mathrm{a}{ }_{-}{ }_{\mathrm{N} c o I} \mathrm{Seq} 8_{\mathrm{XhoI}}$, and pET28a- ${ }_{\text {NoI }}$ CaSM-Seq 8 XhoI. Then, the expression constructs were used to transform competent Escherichia coli BL21 (DE3) cells (Vazyme Co.). For each target protein, several clones were selected and tested by PCR and DNA sequencing to verify the correct insertions of the target genes.

\section{Protein Expression and Purification}

The clones containing the genes that encode for CaSM, CaSMSeq8, and Seq 8 were cultured at $37^{\circ} \mathrm{C}$ overnight in Luria-Bertani broth, containing $1 \mathrm{~mm}$ kanamycin. Then, the overnight grown bacteria were diluted in $1,000 \mathrm{~mL}$ of Luria-Bertani broth (1:100 dilution) and incubated at $37^{\circ} \mathrm{C}$ for $2-4 \mathrm{~h}$, until the $\mathrm{OD}_{600}$ reached 0.6. Then, isopropyl $\beta$-D-1-thiogalactopyranoside was injected into the medium (final concentration: $1 \mathrm{mM}$ ), and the protein expression was induced for another 3-4 h. After centrifugation, the pellets were suspended in a lysis buffer $\left(50 \mathrm{mM} \mathrm{NaH}_{2} \mathrm{PO}_{4}, 300 \mathrm{mM}\right.$ $\mathrm{NaCl}$, and $10 \mathrm{~mm}$ imidazole, $\mathrm{pH}$ 8.0). The cell lysis was enhanced by lysozyme and deoxyribonuclease (Sigma-Aldrich, Burlington, MA, USA), and the samples were centrifuged at $120,000 \mathrm{~g}$ for 20 $\min$ at $4^{\circ} \mathrm{C}$. Finally, the supernatants and pellets were aliquoted and analyzed by SDS-PAGE.

The soluble fractions of the proteins were purified using the Ni-NTA affinity chromatography column (QIAGEN Sciences, Germantown, MD, USA) according to the manufacturer's instructions. Briefly, the columns were equilibrated with the lysis buffer, loaded with the prepared supernatants; then, washed with the washing buffer $\left(50 \mathrm{mM} \mathrm{NaH}_{2} \mathrm{PO}_{4}, 300 \mathrm{mM} \mathrm{NaCl}\right.$, and $30 \mathrm{~mm}$ imidazole, $\mathrm{pH}=8.0$ ); the target proteins were eluted with the elution buffer $\left(50 \mathrm{mM} \mathrm{NaH}_{2} \mathrm{PO}_{4}, 300 \mathrm{mM} \mathrm{NaCl}\right.$, and $250 \mathrm{~mm}$ imidazole, $\mathrm{pH}=8.0$ ). The elutions containing the purified protein were injected into Slide-A-Lyzer ${ }^{\mathrm{TM}}$ Dialysis Cassettes (Thermo Fisher Scientific Inc.) and dialyzed for $12 \mathrm{~h}$ at $4^{\circ} \mathrm{C}$ in phosphate-buffered saline (PBS) to eliminate the influence of ions in the elution buffer and facilitate the VLPs assembly. The purified proteins were analyzed by SDS-PAGE, and their concentrations were determined by Quick Start ${ }^{\mathrm{TM}}$ Bradford Protein Assay Kit (Bio-Rad Laboratories, Inc., Hercules, CA, USA). The purity of the target proteins was evaluated by analyzing the SDS-PAGE gels using the Image J software.

Chimeric Virus-Like Particles Based on the HEV Capsid
Transmission Electron Microscopy

CaSM, CaSM-Seq8, and Seq 8 were diluted to $0.1 \mathrm{mg} / \mathrm{mL}$ and loaded on the carbon-coated grids for $10 \mathrm{~min}$ and negatively stained with $2 \%$ uranyl acetate for another $10 \mathrm{~min}$. The grids were dehydrated and observed by the transmission electron microscope (TEM) F30 (Philips, Amsterdam, The Netherlands). Further, the shape and diameter of the particles on the TEM micrographs were analyzed using the Image-Pro Plus 6.0 software.

\section{Computational Analysis of the Oligomerization States of the}

Target Proteins

The Phyre2 server [29] was used to predict the 3D structures of CaSM, CaSM-Seq8, and Seq8. The structural models were refined using the GalaxyWEB Refine server [30] as previously described [31]. Then, GalaxyWEB Homomer server [32] was used to predict the oligomerization state and the assembly pattern based on the monomer's 3D structures. The predicted models with the highest TM score (structure-based method) or the highest docking score (ab initio docking method) were selected as the optimal models.

\section{Immunomagnetic Separation of Native HEV Virions}

Feces samples containing the native HEV virus (genotype 4, strain NJ703 [GenBank: AY789228]) were stored at $-80^{\circ} \mathrm{C}$ in our lab [33]. The feces supernatant was prepared using PBS. Then, $\mathrm{HEV}$ virions were purified from the feces supernatant by immunomagnetic separation (IMS) using Dynabeads ${ }^{\circledR}$ Protein A (Nanoeast Bio-Technique Co. Ltd., Nanjing, China). The HEV 5G5 monoclonal antibody was used to coat the IMS beads [34], and 1.25 $\mu \mathrm{L}$ Tween-20 and $500 \mu \mathrm{L}$ feces supernatant were added into the coated IMS beads. After the HEV virions were captured by the $5 \mathrm{G} 5$ antibody, the IMS beads were magnetized and washed using $0.05 \%$ Tween-20-PBS. Then, the beads were eluted 3 times using $50 \mu \mathrm{L}$ of $100 \mathrm{~mm}$ glycine buffer $(\mathrm{pH}=2.5)$. The eluant was centrifuged and supplemented with $50 \mu \mathrm{L}$ Tris buffer $(\mathrm{pH}=8.0)$.

\section{Analysis of the Thermal Stability and the Enzymatic}

Proteolysis of the Target Proteins

To study the thermal stability of CaSM, CaSM-Seq8, and Seq8, the samples were diluted to $0.1 \mathrm{mg} / \mathrm{mL}$, aliquoted, and stored at different temperatures $\left(-80^{\circ} \mathrm{C}, 4^{\circ} \mathrm{C}\right.$, and $\left.37^{\circ} \mathrm{C}\right)$. After $1,3,5$, and 7 days post-incubation, the samples were examined by SDS-PAGE to determine the degradation rate.

To explore their resistance to protease digestion, $0.1 \mathrm{mg} / \mathrm{mL}$ of CaSM, CaSM-Seq8, and Seq8 were mixed with an equal volume of simulated gastric fluid (diluted $\mathrm{HCl} 1.64 \mathrm{~mL}$, dd $\mathrm{H}_{2} \mathrm{O} 80 \mathrm{~mL}$, and pepsin $1 \mathrm{~g}$, adjusted with $\mathrm{ddH}_{2} \mathrm{O}$ to $100 \mathrm{~mL}$ ) or simulated intestinal fluid $\left(\mathrm{KH}_{2} \mathrm{PO}_{4} 0.68 \mathrm{~g}\right.$ and trypsin $1 \mathrm{~g}$, adjusted with $\mathrm{NaOH}$ to $\mathrm{pH}=6.8$, total volume $100 \mathrm{~mL}$ ). Then, the samples were incubated at $37^{\circ} \mathrm{C}$ for $2 \mathrm{~h}$ and analyzed by SDS-PAGE.

\section{Antigenicity Analysis}

First, the antigenicity of CaSM, CaSM-Seq8, and Seq8 was evaluated by a computational approach. Since the antigen Seq 8 contains 3 neutralizing epitopes ( 3 different VP1 G-H loops), the exposure of these epitopes in CaSM-Seq8 and Seq 8 was evaluated by the ElliPro server [35]. This server calculates the protrusion index (PI) of each amino acid in a given protein's structural model. The PI values of these epitopes in both CaSM-Seq 8 and Seq 8 were compared by a paired parametric $t$ test. Moreover, the area 
under the PI curve (AUC) of each epitope was calculated as an index evaluating the overall protrusion of each $\mathrm{G}-\mathrm{H}$ loop as a whole.

Next, to experimentally evaluate the antigenicity of these proteins, the immunoreactivity of CaSM, CaSM-Seq8, and Seq8 against anti-FMDV-specific antibodies was tested by an indirect enzyme-linked immunosorbent assay (ELISA) and Western blotting. Since the G-H loops in the Seq 8 antigen were obtained from $\mathrm{O} / \mathrm{HN} / \mathrm{CHA} / 09, \mathrm{O} / \mathrm{IRN} / 2010$, and O/Mya/98 strains, the following anti-FMDV-specific antibodies were used: (1) sera of pigs infected with $\mathrm{O} / \mathrm{Mya} / 98+\mathrm{O} / \mathrm{HN} / \mathrm{CHA} / 09$ strains (sharing the same G-H loops with Seq8); (2) sera of pigs infected with O/ $\mathrm{JMS} / 00+\mathrm{O} / \mathrm{GX} / 09-7$ strains to test the cross-reactivity with different topotypes of FMDV/O serotype; (3) negative sera obtained from FMDV-free pigs; and (4) purified anti-FMDV/O polyclonal antibodies, commercially available for the treatment of FMD. The reactivity difference between the target proteins was statistically assessed by a two-way ANOVA, followed by a Tukey post hoc test.

\section{Mice Immunization and $\operatorname{Ig} G$ Detection}

All animal experiments were performed according to ARRIVE guidelines [36] and in strict accordance with "Care and Use of Laboratory Animals of Southeast University." Welfare evaluations and interventions were performed before, during, and after the experiments.

A total of 40 6-week-old female BALB/c mice were purchased from the Comparative Medicine Center of Yangzhou University. The mice were randomly divided into 4 groups and kept in a pathogen-free environment. CaSM, CaSM-Seq8, and Seq8 (final amount $10 \mu \mathrm{g}$ ) were mixed with the ISA206 adjuvant (SEPPIC, France), and all the mice were immunized by intramuscular injections (group 1: CaSM-Seq8 + ISA206, group 2: Seq8 + ISA206, group 3: $150 \mu \mathrm{L} /$ mouse of the commercial FMDV-inactivated vaccine as a positive control group, and group 4: $150 \mu \mathrm{L} /$ mouse of saline + ISA206 as a negative control group). The commercial FMDV-inactivated vaccine (O/Mya98/XJ/2010 + O/GX/09-7) was bought from Jinyu Baoling Biological Medicine Company (Huhehaote, China). Blood samples were collected from the internal iliac vein before immunization and at weeks $2,4,6,8$, and 10 postimmunization and centrifuged at $12,000 \mathrm{rpm}$ for $20 \mathrm{~min}$. Then, the sera were pooled and stored at $-80^{\circ} \mathrm{C}$. The induced anti-FMDV-specific IgGs in the mice sera were detected by an indirect ELISA. Seq 8 was used as a coating antigen, and HRP-conjugated goat anti-mouse IgG (Thermo Fisher Scientific Inc.) was used as a secondary antibody. The difference in antibody levels was assessed by two-way ANOVA followed by a Tukey post hoc test.

By the end of the study, the mice were euthanized by inhaling a lethal dose of carbon dioxide in the mice asphyxiation chamber (carbon dioxide flowing rate: $2.5 \mathrm{~L} / \mathrm{min}$ ). The mice euthanasia protocol was approved by the Institutional Animal Care and Use Committee of Southeast University.

\section{SDS-PAGE and Western Blotting}

All samples were mixed with an equal volume of $2 \times$ loading buffer (5\% SDS, $20 \%$ glycerol, and $0.002 \%$ bromophenol blue; $1.4 \% \mathrm{v} / \mathrm{v}$ of $\beta$-mercaptoethanol), heated to $100^{\circ} \mathrm{C}$ for $5 \mathrm{~min}$, and then, loaded into a $10-15 \%$ gradient polyacrylamide gel. After the completion of electrophoresis, the gel was stained in a Coomassie staining solution ( $300 \mathrm{~mL}$ methanol, $100 \mathrm{~mL}$ acetic acid, and 1.25 g of Coomassie R-250, adjusted with $\mathrm{ddH}_{2} \mathrm{O}$ to $1,000 \mathrm{~mL}$ ).

For Western blotting, the SDS-PAGE gel without staining was washed with the transfer buffer ( $25 \mathrm{~mm}$ Tris base, $190 \mathrm{~mm}$ glycine, and $20 \% \mathrm{v} / \mathrm{v}$ of methanol, $\mathrm{pH} 8.3$ ). Then, the proteins in the gel were transferred onto the NC membrane at $200 \mathrm{~mA}$ for $2 \mathrm{~h}$ in a transferring cassette (Bio-Rad Laboratories, Inc.). Next, the membrane was incubated in a blocking buffer $(20 \mathrm{mM}$ Tris, $137 \mathrm{mM}$ $\mathrm{NaCl}$, and $0.1 \mathrm{~mL}$ Tween $20, \mathrm{pH}=7.6$, supplemented with $5 \% \mathrm{w} / \mathrm{v}$ of skim milk powder) at $37^{\circ} \mathrm{C}$ for $2 \mathrm{~h}$. The primary antibody was added to the blocking buffer to a final dilution of 1:500, followed by overnight incubation with shaking at $4^{\circ} \mathrm{C}$. The next day, after washing, the membrane was incubated in the blocking buffer containing 1:2,000 of the HRP-conjugated secondary antibody for $2 \mathrm{~h}$ at room temperature. Finally, the reactive bands were developed by the addition of a 3,3'-diaminobenzidine solution.

\section{Indirect ELISAs}

The assays were performed as previously described [37]: (1) 96well flat-bottom plates were coated with the target antigens (200 ng/well) in $1 \mathrm{M}$ Urea-PBS at $4^{\circ} \mathrm{C}$ overnight; (2) after washing with PBS supplemented with $0.5 \%$ Tween 20 (PBST), $100 \mu \mathrm{L}$ of the primary antibody serially diluted $(1: 100,1: 200,1: 400,1: 800,1: 1,600$, $1: 3,200,1: 6,400,1: 12,800$, and $1: 25,600)$ in $1 \%$ casein-PBS was added into the wells and incubated at $37^{\circ} \mathrm{C}$ for $1 \mathrm{~h}$; (3) after washing with PBST, the HRP-conjugated secondary antibody diluted 1:5,000 in $100 \mu \mathrm{L} 1 \%$ casein-PBS was added, and the plates were incubated at $37^{\circ} \mathrm{C}$ for $1 \mathrm{~h}$; (4) after washing with PBST, the plates were developed by the addition of $100 \mu \mathrm{L}$ of a $3,3^{\prime}, 5,5^{\prime}$-tetramethylbenzidine chromogenic substrate; and (5) the reaction was stopped by adding $50 \mu \mathrm{L} 2 \mathrm{M} \mathrm{H}_{2} \mathrm{SO}_{4}$, and after a 20 -min incubation at $37^{\circ} \mathrm{C}$, the absorbance of each well was read at $450 / 630 \mathrm{~nm}$.

\section{Software and Statistical Analysis}

SnapGene 2.3.2 (GSL Biotech) was used for the design, editing, and manipulations of the primers and DNA sequences. PyMOL 2.0.4 (Version 2.0 Schrödinger, LLC.) was used for the visualization of the protein structure models. All the figures were prepared using Adobe Photoshop CC 2019 (Adobe Systems Incorporated), Image J 1.52K (NIH, Bethesda, MD, USA), GraphPad Prism 8 (GraphPad Software, Inc.), and Image-Pro Plus 6.0 (Media Cybernetics, Inc.). Statistical analysis was performed using IBM SPSS V.24 (International Business Machines Corp.). $p \geq 0.05$ was considered as no significance; $0.01 \leq p<0.05$ : ${ }^{*} ; 0.001 \leq p<0.01$ : **; $0.0001 \leq p<0.001:^{* * *} p<0.0001$ : $^{* * * *}$.

\section{Results}

\section{Design and Preparation of the Target Recombinant \\ Proteins}

The target genes (NcoI-CaSM-XhoI, NcoI-CaSMSeq8-XhoI, and NcoI-Seq8-XhoI) were successfully amplified and inserted into the pET28a vector. Then, the constructed plasmids were successfully transformed into competent E. coli BL21 cells. After the selection of the target clones and DNA sequencing, the protein expression 


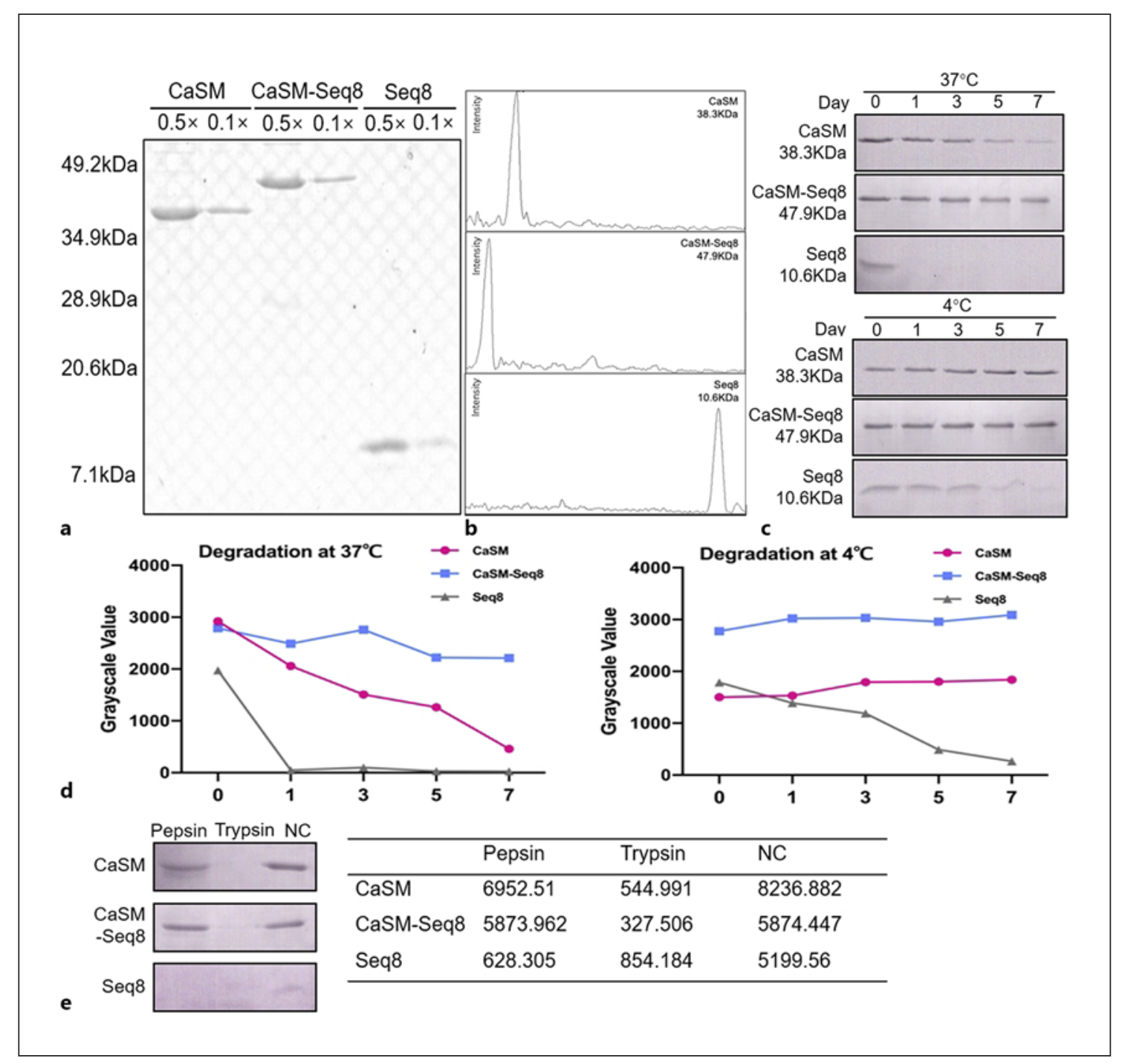

Fig. 1. Preparation and proteolysis analysis of CaSM, CaSM-Seq8, and Seq 8 proteins. SDS-PAGE analysis of 2 dilutions $(\times 0.5$ and $\times 0.1$ ) of the purified recombinant proteins $(\mathbf{a})$; determination of the target proteins purity by the analysis of the SDS-PAGE bands using Image J (b); SDS-PAGE gel showing the thermal stability of the purified proteins at $37^{\circ} \mathrm{C}(\mathbf{c})$ and $4^{\circ} \mathrm{C}$, followed by the grayscale quantification of the target bands (d); SDS-PAGE gel showing the proteolytic action of Pep and Try on CaSM, CaSM-Seq8, and Seq8 (e), followed by a grayscale analysis of the target bands. NC, negative control; Pep, pepsin; Try, trypsin; CaSM, S and M domains of the hepatitis E virus capsid. was induced. The results revealed that CaSM, Seq8, and CaSM-Seq8 were highly overexpressed, and the soluble fractions were successfully purified (Fig. 1a, b).

\section{Assessment of the Thermal Stability and the \\ Proteolysis Resistance}

Physical properties, like thermal stability and resistance to proteolysis, are crucial in vaccine design and development. Therefore, we sought to study whether these properties could be optimized when the Seq8 antigen was presented on CaSM particles. According to the thermal stability results (Fig. 1c), CaSM was stable at $4^{\circ} \mathrm{C}$ and $37^{\circ} \mathrm{C}$, and only a slight degradation occurred after 5 and 7 days of storage at $37^{\circ} \mathrm{C}$. Likewise, CaSM-Seq 8 was also very stable at both $4^{\circ} \mathrm{C}$ and $37^{\circ} \mathrm{C}$. By contrast, Seq 8 was extremely thermolabile with a complete degradation after 1 day at $37^{\circ} \mathrm{C}$ and a gradual degradation at $4^{\circ} \mathrm{C}$ starting from day 1 to day 7 (Fig. 1d). These results indicated that CaSM could indeed enhance the thermal stability of the presented foreign antigen. 


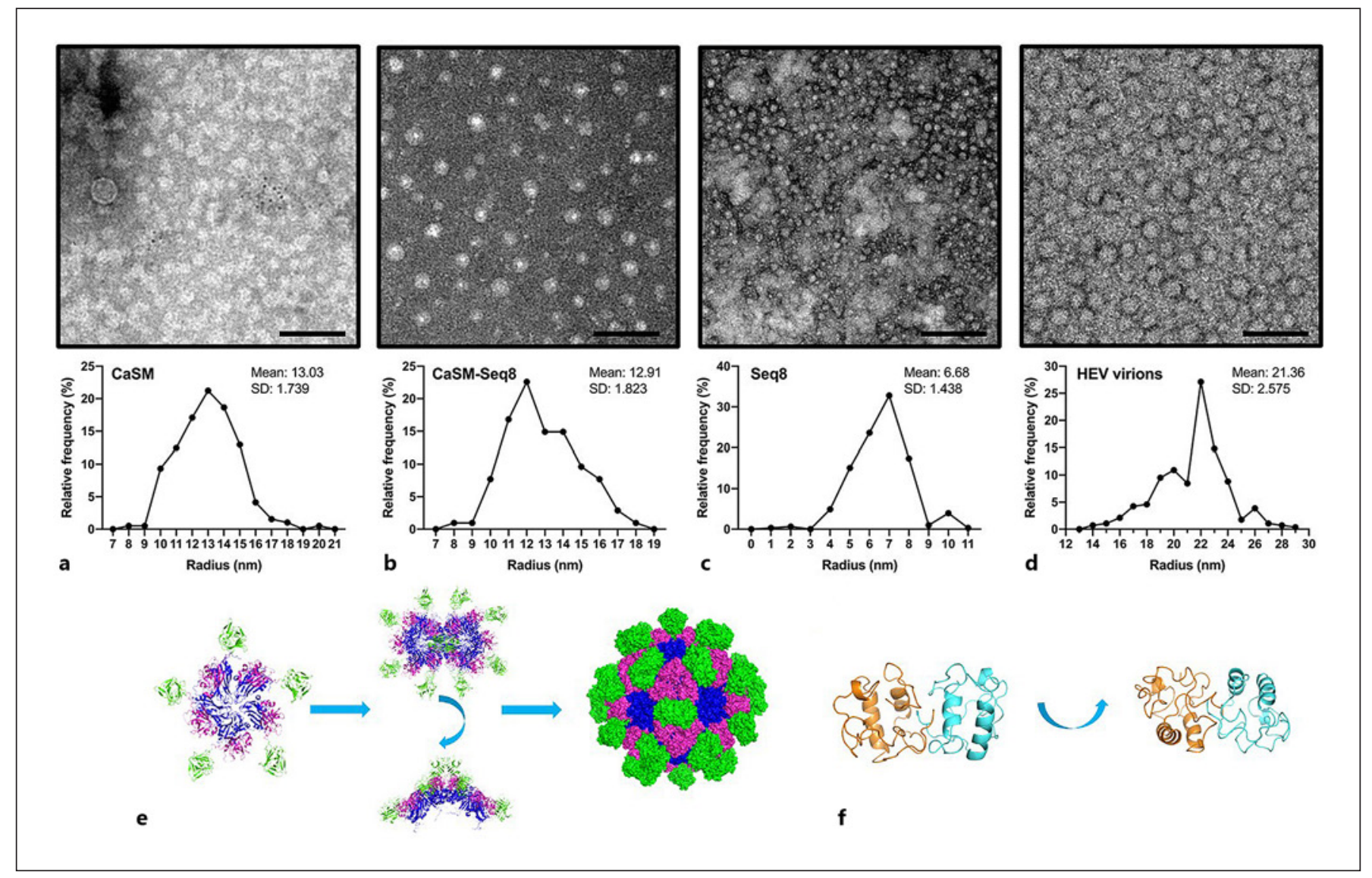

Fig. 2. Morphological analysis of target proteins. a-d Electron micrographs (top) and the radius/frequency analysis (bottom) of CaSM, CaSM-Seq8, Seq8, and native HEV virions, respectively. e The self-assembly patterns of CaSM, CaSM-Seq8, and HEV cap- sid protein (PDB ID: $3 \mathrm{HAG}$ ) as predicted by the GalaxyWEB server. $\mathbf{f}$ The dimerization of Seq8 as predicted by the GalaxyWEB server. CaSM, S and M domains of the hepatitis E virus capsid; HEV, hepatitis $\mathrm{E}$ virus.
Next, we studied whether the carrier CaSM could enhance the resistance of the exogenous antigen to enzymatic digestion (pepsin and trypsin). As shown in Figure 1e, CaSM and CaSM-Seq8 were resistant to pepsin but vulnerable to trypsin. However, Seq 8 was vulnerable to both pepsin and trypsin and was totally digested. This indicated that the CaSM carrier could enhance the resistance of the presented Seq8 antigen to pepsin.

\section{Morphological Assessment of the Particles Formed by} the Target Proteins

The target proteins were visualized under a TEM. Both CaSM and CaSM-Seq8 formed spherically shaped VLPs. According to the radii calculation and frequency analysis, the radius of CaSM was $13.03 \pm 1.739 \mathrm{~nm}$ (Fig. 2a), and the radius of CaSM-Seq8 particles was $12.91 \pm 1.823 \mathrm{~nm}$ (Fig. 2b). However, on the Seq8 micrographs, abundant smaller aggregates (radius: $6.68 \pm 1.438 \mathrm{~nm}$ ) and fewer ir- regular large particles were observed (Fig. 2c), without the classical VLP shape observed with CaSM and CaSM-Seq8. As a control, native HEV virions were also observed, and they displayed a radius of $21.36 \pm 2.575 \mathrm{~nm}$ (Fig. 2d).

To further investigate the reasons for these morphological differences, the GalaxyWEB Homomer server was used to compute the assembly patterns of the studied proteins [32]. The results indicated that CaSM could form pentamers that assembled into decamers, like the native HEV $T=1 / 3$ VLPs (Fig. 2e). Furthermore, CaSM-Seq8 showed the same assembly pattern as CaSM and the native HEV $T=1 / 3$ VLPs (Fig. 2e). However, Seq8 was predicted to form only dimers (Fig. $2 \mathrm{f}$ ), which could explain the observations on the electron micrographs.

\section{Antigenicity Analysis}

First, we sought to assess the antigenicity of Seq 8 and CaSM-Seq8 computationally based on the predicted 3D
42

Intervirology 2022;65:37-48

DOI: $10.1159 / 000515719$
Lu/Behloul/Zhou/Baha/Liu/Wei/Shi/ Meng 


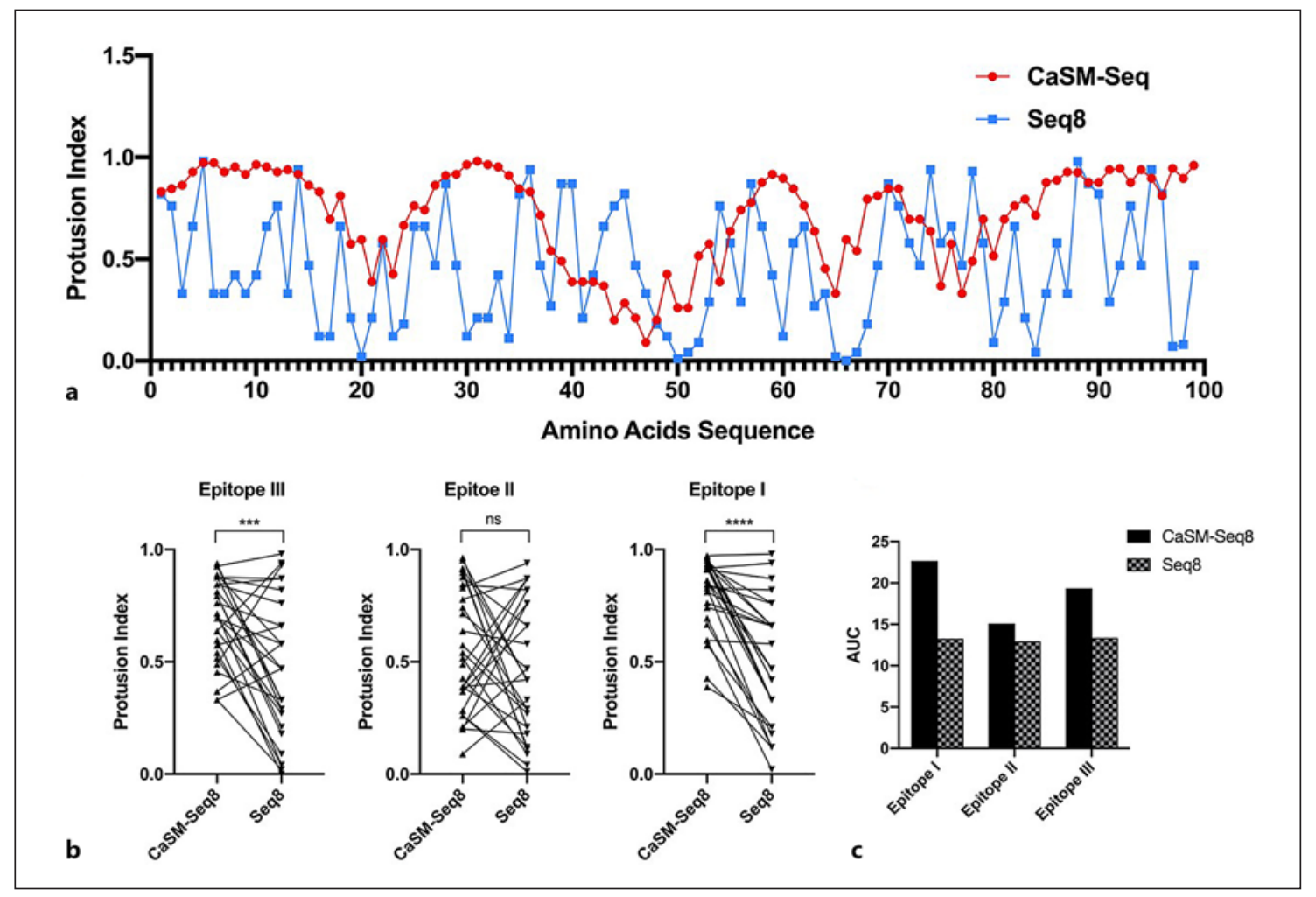

Fig. 3. Computational analysis of the antigenicity of the target proteins. PI of the Seq 8 residues in both CaSM-Seq8 and Seq8 alone (a); comparison of the fluctuation of the PI of the $3 \mathrm{G}-\mathrm{H}$ loops between Seq8 and CaSM-Seq8 (b); column bar representa-

structure models. According to the PI calculation and comparison, most amino acid residues were more exposed in CaSM-Seq8 than in Seq8 (Fig. 3a, b). Since the Seq 8 antigen contains 3 different neutralizing epitopes (3 $\mathrm{G}-\mathrm{H}$ loops), the AUC of each epitope was calculated to evaluate the exposure of each epitope as a whole. The result revealed that the AUCs of epitope I and III were significantly higher in CaSM-Seq8 than in Seq8 (Fig. 3c). All these results suggested that the epitopes were more protruding in CaSM-Seq8 than in Seq8, and thus, the CaSMSeq 8 might have a higher antigenicity than Seq8.

Next, we assessed and compared the antigenicity of CaSM-Seq8 and Seq8 experimentally. The results of the indirect ELISA revealed that at almost all dilutions, the reactivity of CaSM-Seq8 against $\mathrm{O} / \mathrm{Mya} / 98-$ or $\mathrm{O} / \mathrm{HN} /$ CHA/09-induced antibodies (Fig. 4a), O/JMS/00- or O/ GX/09-7-induced antibodies (Fig. 4b), and purified antiFMDV/O polyclonal antibodies (Fig. 4c) was stronger than that of Seq8. Similarly, the Western blotting results revealed that the reactive bands of CaSM-Seq8 had higher grayscales than those of Seq8 (Fig. 4e). Altogether, tion of the AUC of the $3 \mathrm{G}-\mathrm{H}$ loops in Seq8 and CaSM-Seq8 proteins (c). AUC, area under the protrusion index curve; PI, protrusion index; CaSM, S and M domains of the hepatitis E virus capsid. these results were consistent with the computational analysis and indicated an enhanced antigenicity of Seq8 when it was attached to the CaSM protein.

\section{Immunogenicity Analysis}

After the injection of CaSM-Seq8, Seq8, and FMDVinactivated vaccine into mice, the induced FMDV-specific IgG levels were monitored up to 10 weeks postinjection using an indirect ELISA (Fig. 5a). Antibody titers induced by CaSM-Seq 8 were higher than those induced in all the other experimental groups. Moreover, FMDV-specific antibodies appeared earlier in CaSM-Seq8-immunized mice (at week 2) than in the inactivated vaccine group or Seq8-immunized mice (at week 4). Additionally, the humoral response induced by the inactivated vaccine and Seq 8 began to decrease approximately at week 6 and week 8 , respectively. By contrast, the CaSM-Seq8-induced antibodies maintained the highest levels until the end of the experiment. These results indicated that the CaSM platform improved the immunogenicity of Seq8, which is in accordance with the results of the computational analysis 


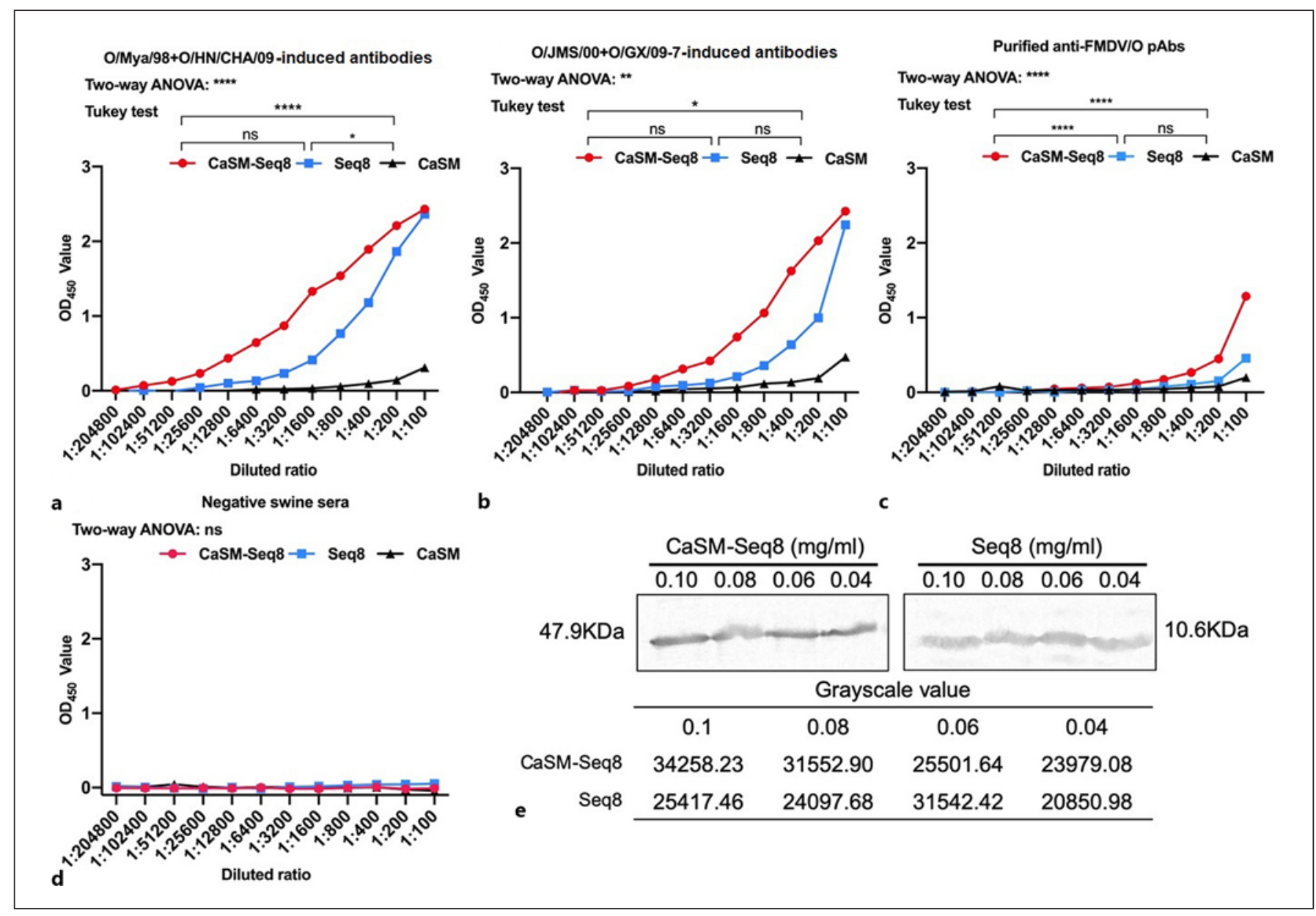

Fig. 4. Experimental evaluation of the antigenicity of the target proteins. a-d Comparison of the immunoreactivity of CaSM, CaSM-Seq8, and Seq8 with FMDV-specific antibodies using indirect ELISA. The anti-FMDV antibodies used in the experiment were $\mathrm{O} / \mathrm{Mya} / 98+\mathrm{O} / \mathrm{HN} / \mathrm{CHA} / 09$-induced antibodies in pigs (a); $\mathrm{O} / \mathrm{JMS} / 00+\mathrm{O} / \mathrm{GX} / 09-7$-induced antibodies in pigs (b); purified

of the antigenicity, where the exposure of the G-H loops was found enhanced by attaching the Seq 8 antigen to CaSM.

\section{Discussion}

HEV, an icosahedral virus with a diameter of approximately $27-32 \mathrm{~nm}$, is an oral-transmitted virus, causing acute viral hepatitis [8]. Its capsid protein is encoded by ORF2 and contains 3 structural domains: $S$ domain (residues 129-319), $\mathrm{M}$ domain (residues 320-455), and $\mathrm{P}$ domain (residues 456-606) which forms protruding spikes outside the VLP (Fig. 5b) [10, 38].
anti-FMDV/O pAbs (c); and negative sera collected from FMDVfree pigs (d); Western blotting analysis with the corresponding grayscale values $(\mathbf{e}){ }^{* * * *} p<0.0001,{ }^{* * *} p<0.001,{ }^{* *} p<0.01,{ }^{*} p<$ 0.05 , and ns: $p>0.05$. CaSM, $\mathrm{S}$ and $\mathrm{M}$ domains of the hepatitis $\mathrm{E}$ virus capsid; FMDV, foot-and-mouth disease virus; pAbs, polyclonal antibodies; ELISA, enzyme-linked immunosorbent assay.

In the present study, the truncated HEV capsid (aa 112-455) named CaSM, comprising the $S$ and $M$ domains as well as the flexible proline-rich hinge linking the $\mathrm{P}$ domain, was selected to present the exogenous antigen Seq8. The selection of this truncated protein was based on the structural features of these domains reported after the elucidation of high-resolution structures of the HEV capsid $[9,10,38]$. The $S$ domain, which is the most conserved region among HEV genotypes [33], formed an icosahedral shell serving as the base for the arrangement of the subsequent domains ( $\mathrm{M}$ and $\mathrm{P}$ ) [38]. Although HEV capsid seemed to share similar folding and morphology with other viruses such as caliciviruses $[39,40]$, the arrangement of the M domain seemed to be unique to the HEV
44

Intervirology 2022;65:37-48

DOI: $10.1159 / 000515719$
Lu/Behloul/Zhou/Baha/Liu/Wei/Shi/ Meng 
Fig. 5. a Immunogenicity analysis by monitoring the induced FMDV-specific antibody titers in mice immunized with CaSMSeq8, Seq8, and the FMDV-inactivated vaccine; ${ }^{* *} p<0.01,{ }^{*} p<0.05$, ns: $p>0.05$. ${ }^{*} p<0.05$, and ns: $p>0.05$. b Structure of the HEV capsid protein ( $\mathrm{S}$ domain in blue, $\mathrm{M}$ domain in purple, and $\mathrm{P}$ domain in green) and its self-assembly into $T=1 \mathrm{VLP}$ (PDB ID: 2ZTN). c Structure of the FMDV capsid protein (PDB ID: 1FOD) with the VP1 G-H loop shown in green (red box) and a schematic of the design of the recombinant antigen Seq8 containing 3 different VP1 G-H loops. d Predicted structure of the constructed CaSM-Seq8. CaSM, S and $\mathrm{M}$ domains of the hepatitis $\mathrm{E}$ virus capsid; FMDV, foot-and-mouth disease virus; VLP, virus-like particle; HEV, hepatitis E virus; ns, no significance.
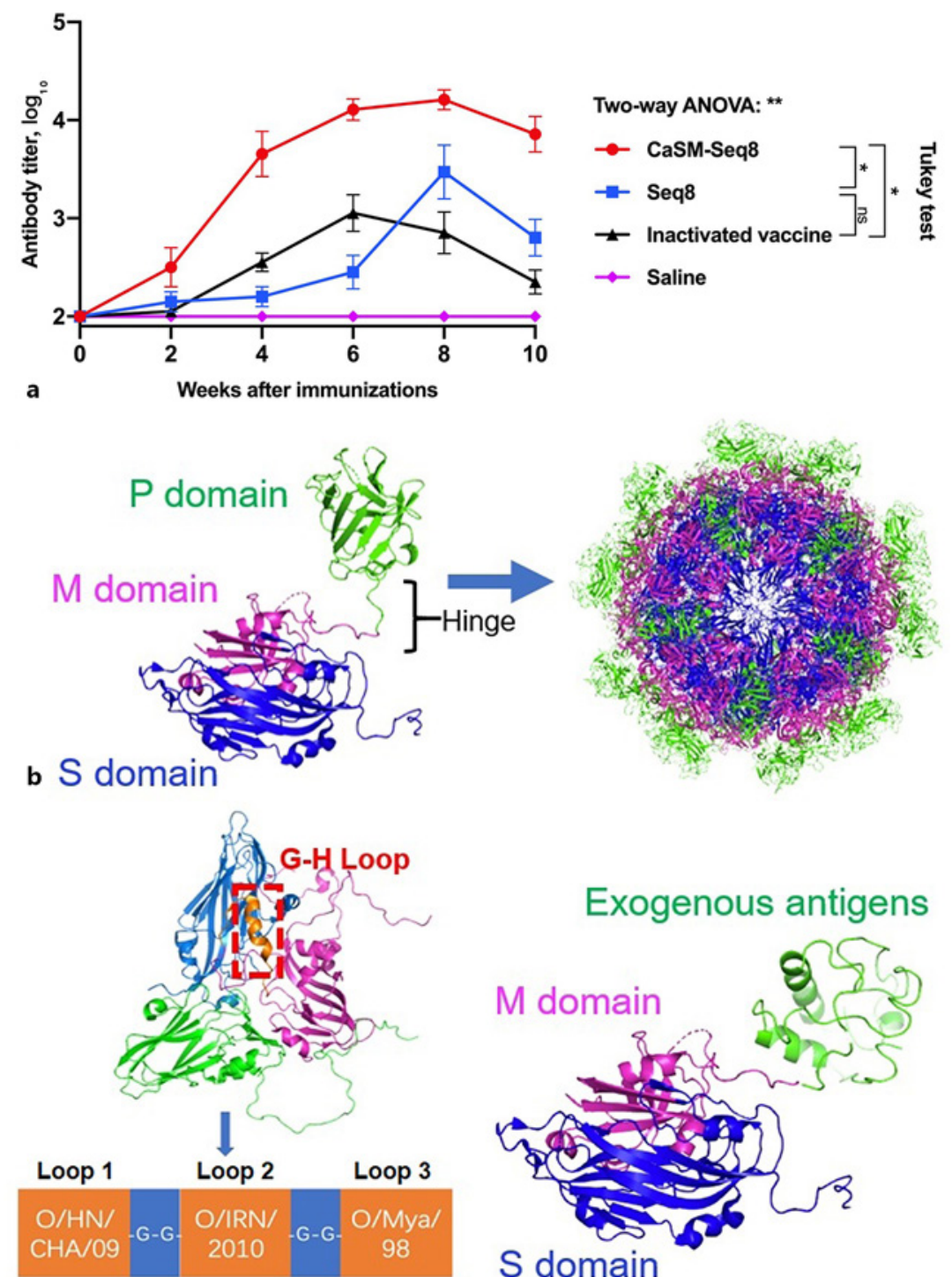

c

Seq8

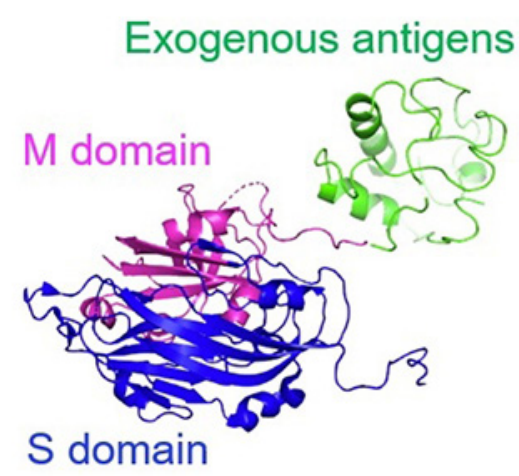

d capsid because its strong interaction with the $S$ domain allows the enhancement of the VLPs stability [38]. Furthermore, unlike the P2 domain of caliciviruses that is inserted into the P1 domain (M domain) [40], the HEV capsid $\mathrm{P}$ domain is independent of the SM-formed shell because of the long proline-rich hinge that links it to the C-terminal of the M domain $[9,10,38]$.

As an exogenous antigen, we designed the Seq 8 chimeric protein (Fig. $5 \mathrm{c}$ ) by combining 3 neutralization epitopes (VP1 G-H loops) derived from 3 different FMDV/O strains as previously reported $[27,28]$. Ac- cording to previous studies, these $\mathrm{G}-\mathrm{H}$ loops elicited neutralizing antibodies and protective immune responses against virulent FMDV strains [24, 26, 28]. However, the VP1 G-H loop had low immunogenicity $[24,26]$, and accordingly, many approaches were adopted to improve the immunogenicity of this peptide, including the incorporation of other T- and B-cell epitopes [25-28]. Therefore, in the present work, we explored the use of HEV CaSM to present Seq8 and the improvement of the immunogenicity of the G-H loops in the chimeric VLPs. In this chimeric construct, the Seq8 antigen was linked to 
the C-terminal of CaSM as a substitute of the P domain in the HEV capsid (Fig. 5d).

Next, through computational analysis, we predicted the oligomerization of the 3 proteins CaSM, CaSM-Seq8, and Seq 8 and found that CaSM and CaSM-Seq 8 could form pentamers and decamers, arranged similarly to those previously described as intermediates in the assembly of HEV VLPs $[10,38,41]$. This suggested that the substitution of the $\mathrm{P}$ domain would not interfere with the proper aggregation of the $S$ and $M$ domains of the CaSMSeq 8 and the formation of the desired chimeric VLPs.

To experimentally confirm these predictions, the 3 target proteins were overexpressed in E. coli, purified, and visualized by TEM. On the micrographs, CaSM self-assembled into VLPs with a diameter of approximately 26 $\mathrm{nm}$, similar in shape but smaller in size than particles observed on the native HEV micrographs. This was expected since the CaSM protein contains the amino acids that previous studies have already identified as essential for truncated HEV ORF2 proteins to form $T=1$ or $T=3$ VLPs, such as aa111, aa126, and aa367 $[12,13]$. More interestingly, the CaSM-Seq8 chimeric protein also formed VLPs with approximately the same diameter and morphology as CaSM particles. It is to note that the VLPs formed by CaSM and CaSM-Seq8 were smaller than the native HEV particles, which is very likely due to the difference in the proteins' size $(38.3 \mathrm{kDa}$ and $47.9 \mathrm{kDa}$ for $\mathrm{CaSM}$ and CaSM-Seq8, respectively, and $70.9 \mathrm{kDa}$ for the native HEV capsid protein). This size parameter could also explain why the Seq 8 antigen alone $(10.6 \mathrm{kDa})$ could not assemble into spherically shaped VLPs similar to the CaSM and CaSM-Seq8 ones. These results along with the computational predictions point toward the conclusion that the substitution of the $\mathrm{P}$ domain with the exogenous Seq8 antigen did not affect the assembly of the HEV S/M domain into particles that present Seq8 as a protruding spike. However, a further determination of the high-resolution structure of CaSM-Seq 8 chimeric VLPs is needed to confirm these observations.

Further, we sought to evaluate whether the formation of the VLPs affected the antigenicity and immunogenicity of the inserted Seq 8 antigen. The bioinformatics analysis revealed that the $3 \mathrm{G}-\mathrm{H}$ loops were more exposed when attached to CaSM than when presented on Seq8 alone, thus suggesting that the antigenicity of Seq 8 would be enhanced in CaSM-Seq8 chimeric VLPs. Indeed, the Western blotting and ELISA results showed that the immunoreactivity of CaSM-Seq8 against FMDV-specific antibodies was more robust than that of Seq 8 alone. Likewise, after injection into mice, like chimeric VLPs, CaSM-
Seq 8 induced higher anti-FMDV IgG levels that appeared earlier, increased faster, and lasted longer than the humoral responses induced by Seq 8 or the FMDV-inactivated vaccine. These results indicated clearly that on one hand, the formed chimeric VLPs did not interfere with the presentation of the Seq8 epitopes, and on the other hand, the optimal exposure of these epitopes enhanced the overall antigenicity/immunogenicity of Seq 8 epitopes. The improved antigenicity and immunogenicity of the Seq 8 moiety in the CaSM-Seq 8 can be explained by (1) the intrinsic characteristics of CaSM-carrier VLPs such as the inclusion of the proline-rich linker that would allow the protrusion of the foreign antigen and augment its conformational flexibility for an optimal interaction with the host immune system (immunogenicity) and the FMDV-specific antibodies (antigenicity); (2) the self-assembly of CaSM-Seq8 into relatively large VLPs (compared to Seq 8 alone) permitted carrying repetitive copies of the target epitopes, which can stimulate more robust immune reactions [42]; and (3) numerous T-cell epitopes have been located in S and $M$ domains [43], which participate in activating a stronger cellular immune response.

Furthermore, it has been previously reported that HEV VLPs could be used as an oral delivery system [6, 16]. Therefore, we investigated the thermal stability and proteolysis resistance of the CaSM-Seq 8 chimeric VLPs. As a result, both properties were enhanced when Seq8 was carried by CaSM particles, thus suggesting that the potential of a CaSM antigen-presenting platform in the design of oral vaccines.

However, further investigation is needed to overcome some of the following limitations of this work: (1) only FMDV Seq 8 was used as the exogenous antigen, and whether CaSM was an excellent VLP platform for other antigens needed further research; (2) high-resolution structures of the CaSM VLPs, CaSM-Seq8 VLPs, and Seq 8 should be determined to study their structural and immunological characteristics at the molecular level; and (3) since many other VLP platforms (HBV, HPV, HIV, etc.) were reported previously [42, 44], a comparative study would be of great interest.

In conclusion, in this study, we demonstrated that the CaSM protein can self-assemble into VLPs, and by substituting only the $\mathrm{P}$ domain with a foreign antigen, the latter was also presented as a protruding domain in the chimeric VLPs, therefore enhancing its antigenicity and immunogenicity. This makes the CaSM particles a potential presentation platform of exogenous immunogens. Furthermore, CaSM-Seq8 investigated here showed promising preliminary results as a chimeric vaccine 
against FMDV that is worth further investigation to evaluate the neutralization activity of CaSM-Seq8-induced antibodies and their in vivo protection against virulent FMDV strains and to select the best adjuvant, the optimal dose, and the best administration route.

\section{Statement of Ethics}

All the animal experiments were approved by the Ethics Committee of the Institutional Animal Care and Use Committee of Southeast University and implemented following the animal experimentation guideline of the Institutional Animal Care and Use Committee of Southeast University and ARRIVE guidelines.

\section{Conflict of Interest Statement}

The authors declare that they have no conflict of interest.

\section{Funding Sources}

This work was supported by the National Natural Science Foundation of China (Grant No. 31770998).

\section{Author Contributions}

J.H.M., T.Y.L., N.B., and R.H.S. conceived and planned the study. T.Y.L., N.B., and Y.Z. performed the analyses and summarized the results. T.Y.L., N.B., Y.Z., S.A., W.J.W., and Z.Z.L. contributed to the interpretation of the results. T.Y.L. and N.B. took the lead in writing the manuscript. All the authors provided critical feedback and helped shape the final version of the manuscript.

\section{Data Availability Statement}

The datasets generated and/or analysed during the current study are available from the corresponding author on reasonable request.

\section{References}

1 Zeltins A. Construction and characterization of virus-like particles: a review. Mol Biotechnol. 2013 Jan;53(1):92-107.

2 Liu J, Dai S, Wang M, Hu Z, Wang H, Deng F. Virus like particle-based vaccines against emerging infectious disease viruses. Virol Sin. 2016 Aug;31(4):279-87.

3 Liu F, Ge S, Li L, Wu X, Liu Z, Wang Z. Viruslike particles: potential veterinary vaccine immunogens. Res Vet Sci. 2012;93(2):553-9.

4 Qi Y, Fan J, Huang W, Zhao C, Wang Y, Kong FT, et al. Expression and characterization of hepatitis E virus-like particles and non-viruslike particles from insect cells. Biotechnol Appl Biochem. 2016 May;63(3):362-70.

5 Ding X, Liu D, Booth G, Gao W, Lu Y. Viruslike particle engineering: from rational design to versatile applications. Biotechnol J. 2018; 13(5):e1700324.

6 Jariyapong P, Xing L, van Houten NE, Li TC, Weerachatyanukul W, Hsieh B, et al. Chimeric hepatitis $\mathrm{E}$ virus-like particle as a carrier for oral-delivery. Vaccine. 2013 Jan 2;31(2): $417-24$.

7 Smith DB, Simmonds P, Izopet J, OliveiraFilho EF, Ulrich RG, Johne R, et al. Proposed reference sequences for hepatitis $\mathrm{E}$ virus subtypes. J Gen Virol. 2016 Mar;97(3):537-42.

8 Nimgaonkar I, Ding Q, Schwartz RE, Ploss A. Hepatitis E virus: advances and challenges. Nat Rev Gastroenterol Hepatol. 2018 Feb; 15(2):96-110.

9 Yamashita T, Mori Y, Miyazaki N, Cheng RH, Yoshimura M, Unno H, et al. Biological and immunological characteristics of hepatitis $\mathrm{E}$ virus-like particles based on the crystal structure. Proc Natl Acad Sci U S A. 2009 Aug 4; 106(31):12986-91.
10 Guu TS, Liu Z, Ye Q, Mata DA, Li K, Yin C, et al. Structure of the hepatitis E virus-like particle suggests mechanisms for virus assembly and receptor binding. Proc Natl Acad Sci U S A. 2009;106(31):12992-7.

11 Li TC, Yamakawa Y, Suzuki K, Tatsumi M, Razak MA, Uchida T, et al. Expression and self-assembly of empty virus-like particles of hepatitis E virus. J Virol. 1997 Oct;71(10): 7207-13.

12 Li TC, Takeda N, Miyamura T, Matsuura Y, Wang JC, Engvall H, et al. Essential elements of the capsid protein for self-assembly into empty virus-like particles of hepatitis E virus. J Virol. 2005 Oct;79(20):12999-3006.

13 Wei M, Zhang X, Yu H, Tang ZM, Wang K, $\mathrm{Li} Z$, et al. Bacteria expressed hepatitis E virus capsid proteins maintain virion-like epitopes. Vaccine. 2014 May 19;32(24):2859-65.

14 Zhang X, Wei M, Pan H, Lin Z, Wang K, Weng $Z$, et al. Robust manufacturing and comprehensive characterization of recombinant hepatitis E virus-like particles in Hecolin $\left({ }^{\circledR}\right)$. Vaccine. 2014;32(32):4039-50.

15 Shima R, Li TC, Sendai Y, Kataoka C, Mori Y, Abe T, et al. Production of hepatitis E viruslike particles presenting multiple foreign epitopes by co-infection of recombinant baculoviruses. Sci Rep. 2016;6:21638.

16 Niikura M, Takamura S, Kim G, Kawai S, Saijo M, Morikawa S, et al. Chimeric recombinant hepatitis $\mathrm{E}$ virus-like particles as an oral vaccine vehicle presenting foreign epitopes. Virology. 2002;293(2):273-80.

17 Kui X, Sun M, Xie T, Wang W, Jiang L, Yan $\mathrm{M}$, et al. The expression, purification, and immunogenicity of a new chimeric virus-like particle. Viral Immunol. 2009;22(1):49-56.
18 Wang CY, Miyazaki N, Yamashita T, Higashiura A, Nakagawa A, Li TC, et al. Crystallization and preliminary X-ray diffraction analysis of recombinant hepatitis $\mathrm{E}$ virus-like particle. Acta Crystallogr Sect F Struct Biol Cryst Commun. 2008;64(4):318-22.

19 Tan M, Jiang X. Recent advancements in combination subunit vaccine development. Hum Vaccin Immunother. 2016;13(1):1805.

$20 \mathrm{Xu} \mathrm{W}$, Zhang Z, Nfon C, Yang M. Genetic and antigenic relationship of foot-and-mouth disease virus serotype $\mathrm{O}$ isolates with the vaccine strain O1/BFS. Vaccine. 2018 Jun 18;36(26): 3802-8.

21 Deepak PR, Saravanan P, Biswal JK, Basagoudanavar SH, Dechamma HJ, Umapathi V, et al. Generation of acid resistant virus like particles of vaccine strains of foot-and-mouth disease virus (FMDV). Biologicals. 2019 Jul; 60:28-35

22 Yamada M, Fukai K, Morioka K, Nishi T, Yamazoe R, Kitano R, et al. Early pathogenesis of the foot-and-mouth disease virus $\mathrm{O} /$ JPN/2010 in experimentally infected pigs. J Vet Med Sci. 2018 Apr 27;80(4):689-700.

23 Zhu Z, Yang F, He J, Li J, Cao W, Li J, et al. First detection of foot-and-mouth disease virus O/ME-SA/Ind2001 in China. Transbound Emerg Dis. 2018 Dec;65(6):2027-31.

24 Brown F. New approaches to vaccination against foot-and-mouth disease. Vaccine. 1992;10(14):1022-6.

25 Wang CY, Chang TY, Walfield AM, Ye J, Shen M, Zhang ML, et al. Synthetic peptidebased vaccine and diagnostic system for effective control of FMD. Biologicals. $2001 \mathrm{Sep}-$ Dec;29(3-4):221-8. 
26 Briand JP, Benkirane N, Guichard G, Newman JF, Van Regenmortel MH, Brown F, et al. A retro-inverso peptide corresponding to the $\mathrm{GH}$ loop of foot-and-mouth disease virus elicits high levels of long-lasting protective neutralizing antibodies. Proc Natl Acad Sci U S A. 1997 Nov 11;94(23):12545-50.

27 Cao Y, Lu Z, Li Y, Sun P, Li D, Li P, et al. Poly (I:C) combined with multi-epitope protein vaccine completely protects against virulent foot-and-mouth disease virus challenge in pigs. Antivir Res. 2013;97(2):145-53.

28 Cao Y, Lu Z, Li D, Fan P, Sun P, Bao H, et al. Evaluation of cross-protection against three topotypes of serotype $\mathrm{O}$ foot-and-mouth disease virus in pigs vaccinated with multi-epitope protein vaccine incorporated with poly (I: C). Vet Microbiol. 2014 Jan 31;168(2-4):294301.

29 Kelley LA, Mezulis S, Yates CM, Wass MN, Sternberg MJ. The Phyre2 web portal for protein modeling, prediction and analysis. Nat Protoc. 2015 Jun;10(6):845-58.

30 Heo L, Park H, Seok C. GalaxyRefine: protein structure refinement driven by side-chain repacking. Nucleic Acids Res. 2013 Jul;41(Web Server issue):W384-8.
31 Wei W, Behloul N, Baha S, Liu Z, Aslam MS, Meng J. Dimerization: a structural feature for the protection of hepatitis $\mathrm{E}$ virus capsid protein against trypsinization. Sci Rep. 2018 Jan 29;8(1):1738.

32 Baek M, Park T, Heo L, Park C, Seok C. GalaxyHomomer: a web server for protein homo-oligomer structure prediction from a monomer sequence or structure. Nucleic Acids Res. 2017 Jul 3;45(W1):W320-W24.

33 Zhai L, Dai X, Meng J. Hepatitis E virus genotyping based on full-length genome and partial genomic regions. Virus Res. 2006 Sep; 120(1-2):57-69.

34 Zhang H, Dai X, Shan X, Meng J. Characterization of antigenic epitopes of the ORF2 protein from hepatitis $\mathrm{E}$ virus genotype 4 . Virus Res. 2009 Jun;142(1-2):140-3.

35 Ponomarenko J, Bui HH, Li W, Fusseder N, Bourne PE, Sette A, et al. ElliPro: a new structure-based tool for the prediction of antibody epitopes. BMC Bioinformatics. 2008;9(1): 514.

36 Kilkenny C, Browne WJ, Cuthill IC, Emerson $\mathrm{M}$, Altman DG. Improving bioscience research reporting: the ARRIVE guidelines for reporting animal research. PLoS Biol. 2010 Jun 29;8(6):e1000412.

37 Liu Z, Behloul N, Baha S, Wei W, Shi R, Meng $J$. Design and immunogenicity analysis of the combined vaccine against zoonotic hepatitis $\mathrm{E}$ and foot-and-mouth disease. Vaccine. 2019 Oct 31;37(46):6922-30.
38 Xing L, Li TC, Mayazaki N, Simon MN, Wall JS, Moore M, et al. Structure of hepatitis E virion-sized particle reveals an RNA-dependent viral assembly pathway. J Biol Chem. 2010 Oct 22;285(43):33175-83.

39 Prasad BV, Hardy ME, Dokland T, Bella J, Rossmann MG, Estes MK. X-ray crystallographic structure of the Norwalk virus capsid. Science. 1999 Oct 8;286(5438):287-90.

40 Chen R, Neill JD, Estes MK, Prasad BV. X-ray structure of a native calicivirus: structural insights into antigenic diversity and host specificity. Proc Natl Acad Sci U S A. 2006 May 23; 103(21):8048-53.

41 Mori Y, Matsuura Y. Structure of hepatitis E viral particle. Virus Res. 2011;161(1):59-64.

42 Grgacic EV, Anderson DA. Virus-like particles: passport to immune recognition. Methods. 2006;40(1):60-5.

43 Li S, Zhang J, Xia N. Lessons from hepatitis E vaccine design. Curr Opin Virol. 2015 Apr;11: 130-6.

44 Naskalska A, Pyrć K. Virus like particles as immunogens and universal nanocarriers. Pol J Microbiol. 2015;64(1):3-13. 